\title{
No-tension bodies: a reinforcement problem
}

\author{
Sara De Faveri ${ }^{\mathrm{a}}$, Lorenzo Freddi ${ }^{\mathrm{b}}$, Roberto Paroni ${ }^{\mathrm{c}}$ \\ ${ }^{a}$ Via Cristofoli, 9. 31015 Conegliano, Treviso, Italy \\ ${ }^{b}$ DIMI, Università di Udine, via delle Scienze 206, 33100 Udine, Italy, email: \\ freddi@dimi. uniud.it \\ ${ }^{c}$ DADU, Università di Sassari, Palazzo del Pou Salit, Piazza Duomo 6, 07041 Alghero, \\ Italy, email: paroni@uniss.it
}

\begin{abstract}
In this work we show that the framework put forward by Lucchesi, Silhavy and Zani [8] to study the equilibrium configurations of panels made of no-tension material can be easily extended to the case of a no-tension material with a reinforcing tensile resistant unidimensional material. This kind of bodies could be used to describe reinforced concrete structures. By solving the equilibrium equations we find a family of solutions each of which is characterized by a singular curve where the stress in the no-tension material concentrates. We show that among these, the curve that minimizes the maximum stress resembles the line tension found experimentally on reinforced concrete beams.
\end{abstract}

Keywords:

No-tension materials, singular stresses, equilibrated tensor fields, reinforced concrete beams

\section{Introduction}

In this work we show that the framework put forward by Lucchesi, Silhavy and Zani [8] to study the equilibrium configurations of panels made of no-tension material, $[5,6,7]$, can be easily extended to the case of a no-tension material with a reinforcing tensile resistant unidimensional material. This kind of bodies could be used to describe reinforced concrete structures.

In $[8,10]$ Lucchesi, Silhavy and Zani look for stress fields that equilibrate the applied loads and are negative semi-definite, so to accomodate the incapability of the material to withstand traction. To simplify the problem they use tensor valued measures to describe the stress field; more precisely, they consider stresses that are tensor valued measures with a divergence which is also a measure, see also [4]. Within this framework the stress field may be singular on some curve, $C_{u}$, to be determined from the equilibrium equations; highly localized stress distributions have been experimentally observed in $[2,3]$. The theory for these generalized stresses has been developed by Lucchesi et al. in $[8,9,10,11,12]$; see also [1]. In particular, the balance of forces is postulated only in a weak 
form that allows to take into account the singularities of the stress in a simple and direct way.

To model a no-tension body with a unidimensional reinforcement we prescribe, in the reference configuration, a fixed curve $C_{r}$ representing the region occupied by the reinforcement. On this curve the stress field will be allowed to be singular and to be positive definite in order to model the fact that the reinforcement could support traction forces.

While in the reinforcement the only unknown is the stress, since $C_{r}$ is a priori given, in the singular curve within the no-tension material the unknowns are the stress and the curve $C_{u}$ itself. Besides these, also the density with respect to the Lebesgue measure of the stress field in the no-tension material is unknown.

More precisely, we deal with a body occupying a region $U$ that is divided by a singular "curve" $C=C_{u} \cup C_{r}$ and the stress $\mathbb{T}$ is a measure that is the sum of an absolutely continuous part w.r.t. the Lebesgue measure on $U \backslash C$, with density $\mathbb{T}_{a}$, and a measure concentrated on $C$. Denoting by $\mathbb{T}_{r}$ and $\mathbb{T}_{u}$ the density of the measures concentrated on $C_{r}$ and $C_{u}$, respectively, we require that $\mathbb{T}_{a}$ and $\mathbb{T}_{u}$ are negative semi-definite, since they represents the stress in the no-tension material while $\mathbb{T}_{r}$ could be positive. Therefore, the unknowns of the problem are $\mathbb{T}_{a}, \mathbb{T}_{r}, \mathbb{T}_{u}$ and $C_{u}$.

In Section 2, after recalling one of the main results of [8], we derive the equilibrium equations from the balance equations in the weak form for the case of a rectangular panel with a straight horizontal reinforcement. The choice of this configuration is motivated by the fact that it describes the geometry of reinforced concrete beams. In Section 3 we study the equilibrium problem for the structure subjected to a uniform load on the top of the panel and clamped on two intervals at its basis. Since we are considering only the equilibrium equations, we find that the solution is not unique. In fact, we find a family of solutions each of which is characterized by a singular curve $C_{u}$. We show that the curve that minimizes the maximum stress in the no-tension material, which we call optimal singular curve, resembles the line tension found experimentally on reinforced concrete beams.

\section{Equilibrated tensor fields and balance equations}

In this section we briefly recall the basic notion of equilibrated tensor field and the corresponding balance equations using the notation of [8], see also [10, 14]. To the same paper, and to the references therein, we refer for a complete and detailed presentation.

Let $U$ be an open subset of $\mathbb{R}^{n}$ and $\partial U$ its topological boundary. Let $V$ be a finite-dimensional real inner product space. We denote by $M(U, V)$ and $M(\partial U, V)$ the set of $V$-valued Borel measures supported on $U$ and $\partial U$, respectively. By Lin we denote the space of all linear transformations (tensors) from $\mathbb{R}^{n}$ into $\mathbb{R}^{n}$ with the Euclidean inner product.

Definition 1. A tensor-valued measure $T \in \mathrm{M}(U, \operatorname{Lin})$ is said to be an equilibrated tensor field if there exist measures (actually unique) $b_{0} \in \mathrm{M}\left(U, \mathbb{R}^{n}\right)$ and 
$t_{0} \in \mathrm{M}\left(\partial U, \mathbb{R}^{n}\right)$ such that

$$
\int_{U} \nabla \varphi \cdot d T=\int_{U} \varphi \cdot d b_{0}+\int_{\partial U} \varphi \cdot d t_{0}
$$

for each $\varphi \in C_{c}^{\infty}\left(\mathbb{R}^{n} ; \mathbb{R}^{n}\right)$. The pair $\left(b_{0}, t_{0}\right)$ is called the load corresponding to $T$.

It follows that, the distributional divergence of an equilibrated tensor field $T$ is a vector measure $\operatorname{div} T \in M\left(U ; \mathbb{R}^{n}\right)$. By using (1) we can see that the map

$$
\langle\mathrm{N}(T), \varphi\rangle:=\int_{U} \nabla \varphi \cdot d T+\int_{U} \varphi \cdot d \operatorname{div} T, \quad \varphi \in C_{c}^{\infty}\left(\mathbb{R}^{n}, \mathbb{R}^{n}\right)
$$

is a measure concentrated on $\partial U$, which is called the normal trace of $T$ at the boundary; hence

$$
\langle\mathrm{N}(T), \varphi\rangle=\int_{\partial U} \varphi \cdot d \mathrm{~N}(T)
$$

and the following Green's formula

$$
\int_{U} \nabla \varphi \cdot d T=-\int_{U} \varphi \cdot d \operatorname{div} T+\int_{\partial U} \varphi \cdot d \mathrm{~N}(T)
$$

holds for every $\varphi \in C_{c}^{\infty}\left(\mathbb{R}^{n}, \mathbb{R}^{n}\right)$.

By comparing (1) and (3) we obtain that any equilibrated tensor field satisfies the balance equations

$$
\left\{\begin{array}{l}
-\operatorname{div} T=b_{0}, \\
\mathrm{~N}(T)=t_{0} .
\end{array}\right.
$$

Actually, this is the set of equilibrium equations for a continuous body under the action of a body force given by a prescribed measure $b_{0} \in \mathrm{M}\left(U, \mathbb{R}^{n}\right)$ and a boundary traction given by a prescribed measure $t_{0} \in \mathrm{M}\left(\partial U, \mathbb{R}^{n}\right)$. In particular, if $b_{0}$ is absolutely continuous with respect to the Lebesgue measure then $\operatorname{div} T$ must be absolutely continuous as well.

\subsection{A reinforced panel}

In [8], Lucchesi, Šilavý and Zani, after having developed the theory of equilibrated stress fields for no-tension bodies, have studied several two dimensional equilibrium problems for multi-rectangular panels.

Inspired by their work, we study the statics of a two dimensional rectangular panel made of no-tension material and reinforced by means of a straight unidimensional continuum capable to resist also to traction forces. This system models, for instance, a reinforced concrete beam.

We denote by $U$ the rectangular region occupied by the panel in its reference configuration and by $C_{r} \subset U$ the uni-dimensional straight line occupied by the reinforcing material. We assume $C_{r}$ to be parallel to the basis of $U$. Since the panel is made of no-tension material, the stress in $U \backslash C_{r}$ is assumed to be symmetric and negative semidefinite. 


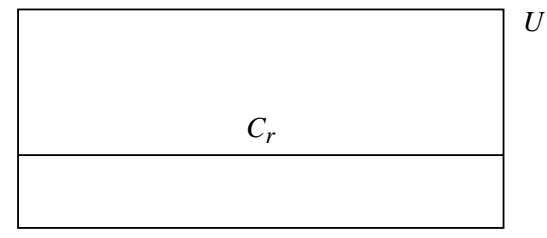

Figure 1: a concrete panel reinforced by a straight unidimensional continuum made of tensile resistant material

In the next section we consider an equilibrium problem under prescribed loads and constraints and look for solutions among equilibrated tensor fields $\mathbb{T}$, which are actually solutions to the equilibrium equations (4).

Following the ideas of [8], in this paper we are going to search only special solutions of (4) by restricting the set of admissible stress tensor fields to those $\mathbb{T}$ whose singularities concentrate on $C_{r}$ and along a simple piecewise smooth curve $C_{u}$ (like for instance in Figure 2 where $C_{u}=C_{1} \cup C_{2} \cup C_{3}$ ) with endpoints $h_{1}$ and $h_{2}$ on the boundary of $U$ and disjoint from the endpoints $e_{1}$ and $e_{2}$ of $C_{r}$.

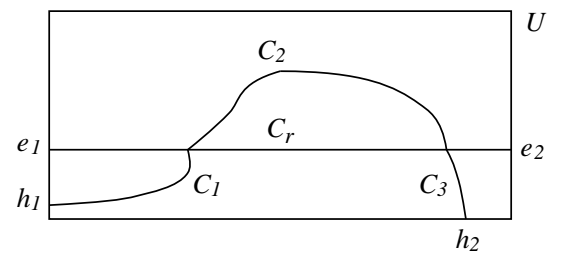

Figure 2: we allow $\mathbb{T}$ to be singular on $C_{r}$ and along an unknown simple piecewise smooth curve $C_{u}=C_{1} \cup C_{2} \cup C_{3}$

This means that the stress field $\mathbb{T}$ is assumed to be the sum of a measure absolutely continuous with respect to the Lebesgue's measure with a smooth density $\mathbb{T}_{a}$ in $U \backslash\left(C_{u} \cup C_{r}\right)$ which has a continuous extension, again denoted by $\mathbb{T}_{a}$, with $\mathscr{L}^{2}$-integrable derivative, to the closure of any connected component of $U \backslash\left(C_{u} \cup C_{r}\right)$ (hence, in particular, $\mathscr{H}^{1}$-integrable on $\partial U$ ) and two measures concentrated on $C_{r}$ and $C_{u}$ whose densities are piecewise smooth superficial tensor fields $\mathbb{T}_{r}$ and $\mathbb{T}_{u}$, respectively, that is

$$
\mathbb{T}_{r}=\sigma_{r}(s) \mathrm{t}_{r}(s) \otimes \mathrm{t}_{r}(s), \quad \mathbb{T}_{u}=\sigma_{u}(s) \mathrm{t}_{u}(s) \otimes \mathrm{t}_{u}(s),
$$

where $\sigma_{r}$ and $\sigma_{u}$ are piecewise smooth scalar fields, respectively, on $C_{r}$ and $C_{u}$ (that is they are allowed to jump only on the intersection points of the two curves with finite right and left limits in such points and have $\mathscr{H}^{1}$-integrable derivative), $s$ is the arclength and $\mathrm{t}_{r}(s), \mathrm{t}_{u}(s)$ denote the respective tangent 
unit vectors. Summarizing, the unknowns of the problem are the curve $C_{u}$, the tensor field $\mathbb{T}_{a}$ and the scalar fields $\sigma_{r}$ and $\sigma_{u}$.

The admissible stress tensor fields are then of the form

$$
\mathbb{T}:=\mathbb{T}_{a} \mathscr{L}^{2}\left\lfloor U+\mathbb{T}_{r} \mathscr{H}^{1}\left\lfloor C_{r}+\mathbb{T}_{u} \mathscr{H}^{1}\left\lfloor C_{u}\right.\right.\right.
$$

and, as shown in [8, Proposition 1], they are equilibrated. Therefore, once having defined the applied loads, the equilibrium equations are given by (4). To make them more explicit we compute $\operatorname{div} \mathbb{T}$ and $N(\mathbb{T})$. It will be shown that

$$
\begin{aligned}
\operatorname{div} \mathbb{T}= & \operatorname{div} \mathbb{T}_{a} \mathscr{L}^{2}\lfloor U \\
& +\left(\frac{d}{d s}\left(\sigma_{r} \mathrm{t}_{r}\right)-\left[\mathbb{T}_{a}\right] \mathrm{n}_{r}\right) \mathscr{H}^{1}\left\lfloor C_{r}\right. \\
& +\left(\frac{d}{d s}\left(\sigma_{u} \mathrm{t}_{u}\right)-\left[\mathbb{T}_{a}\right] \mathrm{n}_{u}\right) \mathscr{H}^{1}\left\lfloor C_{u}\right. \\
& +\sum_{j=1}^{p}\left[\sigma_{r}\right] \mathrm{t}_{r} \delta_{c_{j}}+\sum_{j=1}^{p}\left[\sigma_{u} \mathrm{t}_{u}\right] \delta_{c_{j}}
\end{aligned}
$$

and

$$
\mathrm{N}(\mathbb{T})=\mathbb{T}_{a} \mathrm{~m} \mathscr{H}^{1}\left\lfloor\partial U+\sigma_{r} \mathrm{t}_{r}\left(\delta_{e_{2}}-\delta_{e_{1}}\right)+\sigma_{u} \mathrm{t}_{u}\left(\delta_{h_{2}}-\delta_{h_{1}}\right)\right.
$$

where $\left(\mathrm{t}_{r}, \mathrm{n}_{r}\right)$ and $\left(\mathrm{t}_{u}, \mathrm{n}_{u}\right)$ are unit tangent and normal vectors to $C_{r}$ and $C_{u}, \mathrm{~m}$ is the outer normal to $\partial U$ and $c_{j}(j=1, \ldots, p)$ are the intersection points between the two curves. Moreover, $\left[\mathbb{T}_{a}\right]$ denotes the jump of $\mathbb{T}_{a}$ across the curves, while $\left[\sigma_{r} \mathrm{t}_{r}\right](P)$ and $\left[\sigma_{u} \mathrm{t}_{u}\right](P)$ are the jumps of $\sigma_{r}$ and $\sigma_{u}$ at the point $P$ along $C_{r}$ and $C_{u}$. The jumps are evaluated according to the orientation defined, either by the normal or the tangent vectors. Hence, for instance, $\left[\sigma_{u} \mathrm{t}_{u}\right] \delta_{c_{1}}$ simply means $\left[\sigma_{u} \mathrm{t}_{u}\right]\left(c_{1}\right):=\sigma_{u}\left(s\left(c_{1}\right)^{+}\right) \mathrm{t}_{u}\left(s\left(c_{1}\right)^{+}\right)-\sigma_{u}\left(s\left(c_{1}\right)^{-}\right) \mathrm{t}_{u}\left(s\left(c_{1}\right)^{-}\right)$, where + and - denote the right and left limits referred to the chosen parametrization $s$.

To simplify the computations, we confine ourselves to the case $p=2$; that is $C_{r} \cap C_{u}=\left\{c_{1}, c_{2}\right\} \subset U$. In this case there are two possible situations: one in which the two endpoints of $C_{u}$ are both below $C_{r}$ as in Figure 2, and the other in which they are both above. To fix ideas we suppose to be in the first situation.

The domain $U$ turns out to be divided in 5 parts called $U_{i}, i=1,2,3,4,5$, according to Figure 3 .

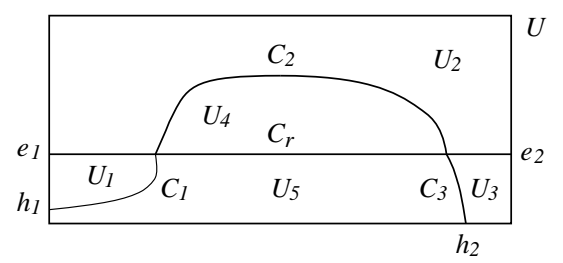

Figure 3: the five regions $U_{i}, i=1,2,3,4,5$ 
Recalling the expression (6) of $\mathbb{T}$, for any $\varphi \in C_{c}\left(\mathbb{R}^{2} ; \mathbb{R}^{2}\right)$ we have

$$
\begin{aligned}
\int_{U} \nabla \varphi \cdot d \mathbb{T}= & \int_{U} \nabla \varphi \cdot \mathbb{T}_{a} d \mathscr{L}^{2}+\int_{C_{r}} \nabla \varphi \cdot \mathbb{T}_{r} d \mathscr{H}^{1} \\
& +\int_{C_{u}} \nabla \varphi \cdot \mathbb{T}_{u} d \mathscr{H}^{1} .
\end{aligned}
$$

After denoting by $\mathbb{T}_{a}^{i}$ the trace of $\mathbb{T}_{a}$ on $\partial U_{i}$ and $\mathrm{n}^{i}$ the corresponding outer normal, the first term on the right-hand side of (9) becomes

$$
\begin{aligned}
& \int_{U} \nabla \varphi \cdot \mathbb{T}_{a} d \mathscr{L}^{2}= \\
& =-\sum_{i=1}^{5} \int_{U_{i}} \varphi \cdot \operatorname{div} \mathbb{T}_{a} d x+\sum_{i=1}^{5} \int_{\partial U_{i}} \varphi \cdot \mathbb{T}_{a}^{i} \mathrm{n}^{i} d s \\
& =-\int_{U} \varphi \cdot \operatorname{div} \mathbb{T}_{a} d x+\int_{\partial U} \varphi \cdot \mathbb{T}_{a} \mathrm{~m} d s \\
& +\sum_{i, j=1, i<j}^{5} \int_{\partial U_{i} \cap \partial U_{j}} \varphi \cdot\left(\mathbb{T}_{a}^{i}-\mathbb{T}_{a}^{j}\right) \mathrm{n}^{i} d s \\
& =-\int_{U} \varphi \cdot \operatorname{div} \mathbb{T}_{a} d x+\int_{\partial U} \varphi \cdot \mathbb{T}_{a} \mathrm{~m} d s \\
& +\int_{C_{r}} \varphi \cdot\left[\mathbb{T}_{a}\right] \mathrm{n}_{r} d s+\int_{C_{u}} \varphi \cdot\left[\mathbb{T}_{a}\right] \mathrm{n}_{u} d s,
\end{aligned}
$$

where

$$
\left[\mathbb{T}_{a}\right] \mathrm{n}_{r}= \begin{cases}\left(\mathbb{T}_{a}^{1}-\mathbb{T}_{a}^{2}\right) \mathrm{n}^{1} & \text { on } \partial U_{1} \cap \partial U_{2}, \\ \left(\mathbb{T}_{a}^{2}-\mathbb{T}_{a}^{3}\right) \mathrm{n}^{2} & \text { on } \partial U_{2} \cap \partial U_{3}, \\ \left(\mathbb{T}_{a}^{4}-\mathbb{T}_{a}^{5}\right) \mathrm{n}^{4} & \text { on } \partial U_{4} \cap \partial U_{5},\end{cases}
$$

and

$$
\left[\mathbb{T}_{a}\right] \mathrm{n}_{u}= \begin{cases}\left(\mathbb{T}_{a}^{1}-\mathbb{T}_{a}^{5}\right) \mathrm{n}^{1} & \text { on } \partial U_{1} \cap \partial U_{5}=C_{1}, \\ \left(\mathbb{T}_{a}^{2}-\mathbb{T}_{a}^{4}\right) \mathrm{n}^{2} & \text { on } \partial U_{2} \cap \partial U_{4}=C_{2}, \\ \left(\mathbb{T}_{a}^{3}-\mathbb{T}_{a}^{5}\right) \mathrm{n}^{3} & \text { on } \partial U_{3} \cap \partial U_{5}=C_{3} .\end{cases}
$$

To make the computation of the second term on the right-hand side of (9) easier, we introduce a positive orientation on the curve $C_{r}$ in which $e_{1}$ is the initial endpoint (hence $e_{2}$ is the final endpoint). By using the fact that $\left(\mathrm{t}_{r} \otimes \mathrm{t}_{r}\right)$. $\nabla\left(\varphi \circ \gamma_{r}\right)=\mathrm{t}_{r} \cdot \frac{d}{d s}\left(\varphi \circ \gamma_{r}\right)$ where $\gamma_{r}(s)$ is any regular parametrization of $C_{r}$ and 
recalling the expression (5) of $\mathbb{T}_{r}$, we have

$$
\begin{aligned}
& \int_{C_{r}} \nabla \varphi \cdot \mathbb{T}_{r} d \mathscr{H}^{1}= \\
&=\int_{\partial U_{1} \cap \partial U_{2}} \sigma_{r}\left(\mathrm{t}_{r} \otimes \mathrm{t}_{r}\right) \cdot \nabla \varphi d s+\int_{\partial U_{2} \cap \partial U_{3}} \sigma_{r}\left(\mathrm{t}_{r} \otimes \mathrm{t}_{r}\right) \cdot \nabla \varphi d s \\
& \quad+\int_{\partial U_{4} \cap \partial U_{5}} \sigma_{r}\left(\mathrm{t}_{r} \otimes \mathrm{t}_{r}\right) \cdot \nabla \varphi d s \\
&=-\int_{C_{r}} \frac{d}{d s}\left(\sigma_{r} \mathrm{t}_{r}\right) \cdot \varphi d s \\
&+\sigma_{r}\left(c_{1}^{-}\right) \mathrm{t}_{r}\left(c_{1}\right) \cdot \varphi\left(c_{1}\right)-\sigma_{r}\left(e_{1}\right) \mathrm{t}_{r}\left(e_{1}\right) \cdot \varphi\left(e_{1}\right) \\
&+\sigma_{r}\left(c_{2}^{-}\right) \mathrm{t}_{r}\left(c_{2}\right) \cdot \varphi\left(c_{2}\right)-\sigma_{r}\left(c_{1}^{+}\right) \mathrm{t}_{r}\left(c_{1}\right) \cdot \varphi\left(c_{1}\right) \\
&+\sigma_{r}\left(e_{2}\right) \mathrm{t}_{r}\left(e_{2}\right) \cdot \varphi\left(e_{2}\right)-\sigma_{r}\left(c_{2}^{+}\right) \mathrm{t}_{r}\left(c_{2}\right) \cdot \varphi\left(c_{2}\right) \\
&=-\int_{C_{r}} \frac{d}{d s}\left(\sigma_{r} \mathrm{t}_{r}\right) \cdot \varphi d s-\int\left[\sigma_{r}\right] \mathrm{t}_{r} \cdot \varphi d\left(\delta_{c_{1}}+\delta_{c_{2}}\right) \\
&+\int \sigma_{r} \mathrm{t}_{r} \cdot \varphi d\left(\delta_{e_{2}}-\delta_{e_{1}}\right) .
\end{aligned}
$$

Analogously, having fixed on $C_{u}$ a positive orientation which goes from $h_{1}$ to $h_{2}$, we have

$$
\begin{aligned}
\int_{C_{u}} & \nabla \varphi \cdot \mathbb{T}_{u} d \mathscr{H}^{1}= \\
= & -\int_{C_{u}} \frac{d}{d s}\left(\sigma_{u} \mathrm{t}_{u}\right) \cdot \varphi d s-\int\left[\sigma_{u} \mathrm{t}_{u}\right] \cdot \varphi d\left(\delta_{c_{1}}+\delta_{c_{2}}\right) \\
& +\int \sigma_{u} \mathrm{t}_{u} \cdot \varphi d\left(\delta_{h_{2}}-\delta_{h_{1}}\right) .
\end{aligned}
$$

By putting all togheter we find

$$
\begin{aligned}
\int_{U} \nabla \varphi \cdot d \mathbb{T}= & -\int_{U} \varphi \cdot \operatorname{div} \mathbb{T}_{a} d x+\int_{\partial U} \varphi \cdot \mathbb{T}_{a} \mathrm{~m} d \mathscr{H}^{1} \\
& +\int_{C_{r}}\left(\left[\mathbb{T}_{a}\right] \mathrm{n}_{r}-\frac{d}{d s}\left(\sigma_{r} \mathrm{t}_{r}\right)\right) \cdot \varphi d \mathscr{H}^{1} \\
& +\int_{C_{u}}\left(\left[\mathbb{T}_{a}\right] \mathrm{n}_{u}-\frac{d}{d s}\left(\sigma_{u} \mathrm{t}_{u}\right)\right) \cdot \varphi d \mathscr{H}^{1} \\
& -\int\left(\left[\sigma_{r}\right] \mathrm{t}_{r}+\left[\sigma_{u} \mathrm{t}_{u}\right]\right) \cdot \varphi d\left(\delta_{c_{1}}+\delta_{c_{2}}\right) \\
& +\sigma_{r} \mathrm{t}_{r} \cdot \varphi d\left(\delta_{e_{2}}-\delta_{e_{1}}\right) \\
& +\int \sigma_{u} \mathrm{t}_{u} \cdot \varphi d\left(\delta_{h_{2}}-\delta_{h_{1}}\right) .
\end{aligned}
$$

The claimed expression for $\operatorname{div} \mathbb{T}$ is then obtained by taking test functions $\varphi \in$ 
$C_{c}^{\infty}\left(U ; \mathbb{R}^{2}\right)$ and using the fact that, within this choice,

$$
\int_{U} \varphi \cdot d \operatorname{div} \mathbb{T}=-\int_{U} \nabla \varphi \cdot d \mathbb{T} .
$$

After that, $\mathbb{N}(\mathbb{T})$ is easily computed by means of (2).

\section{The equilibrium problem}

In this section we assume the panel to be subjected to boundary loads only and to be clamped on two regions $V_{1}$ and $V_{2}$ of width $d$ contained in the lower basis.

It is useful to introduce an orthogonal coordinate system $(x, y)$ with the origin in the middle point of the lower side of the panel, with the $x$ axis pointing right and the $y$ axis pointing upward (see Figure 4); let $\left(\overline{\mathrm{e}}_{1}, \overline{\mathrm{e}}_{2}\right)$ be the associated canonical basis. In this reference we take $U=(-b / 2, b / 2) \times(0, h)$, $V_{1}=(-b / 2,-b / 2+d) \times\{0\}, V_{2}=(b / 2-d, b / 2) \times\{0\}$ with $b, h>0$ and $d \in(0, b / 2)$.

We assume that the system is subjected to a vertical load, $-p_{0} \overline{\mathrm{e}}_{2}$, distributed on its upper side, $y=h$. The couple of measures describing the loads is then given by

$$
b_{0}=0, \quad t_{0}=-p_{0} \overline{\mathrm{e}}_{2}\left\lfloor\Gamma+\Phi\left\lfloor V_{1} \cup V_{2}\right.\right.
$$

where $\Gamma=(-b / 2, b / 2) \times\{h\}$ is the upper side of the panel and $V_{1}$ and $V_{2}$ are the subsets of the lateral boundary of $U$ in which the body is clamped and $\Phi\left\lfloor V_{1} \cup V_{2}\right.$ is a vector valued measure representing the reaction of the constraint and which is a-priori unknown.

Thanks to (7) and (8), the equilibrium equations (4) rewrite

$$
\left\{\begin{array}{l}
\operatorname{div}_{a} \mathbb{L}_{a}\left\lfloor U+\left(\frac{d}{d s}\left(\sigma_{r} \mathrm{t}_{r}\right)-\left[\mathbb{T}_{a}\right] \mathrm{n}_{r}\right) \mathscr{H}^{1}\left\lfloor C_{r}+\right.\right. \\
\quad+\left(\frac{d}{d s}\left(\sigma_{u} \mathrm{t}_{u}\right)-\left[\mathbb{T}_{a}\right] \mathrm{n}_{u}\right) \mathscr{H}^{1}\left\lfloor C_{u}\right. \\
\quad+\sum_{j=1}^{2}\left[\sigma_{r}\right] \mathrm{t}_{r} \delta_{c_{j}}+\sum_{j=1}^{2}\left[\sigma_{u} \mathrm{t}_{u}\right] \delta_{c_{j}}=0, \\
\mathbb{T}_{a} \mathrm{~m} \mathscr{H}^{1}\left\lfloor\partial U+\sigma_{r} \mathrm{t}_{r}\left(\delta_{e_{2}}-\delta_{e_{1}}\right)+\sigma_{u} \mathrm{t}_{u}\left(\delta_{h_{2}}-\delta_{h_{1}}\right)=\right. \\
=-p_{0} \overline{\mathrm{e}}_{2}\left\lfloor\Gamma+\Phi\left\lfloor V_{1} \cup V_{2},\right.\right.
\end{array}\right.
$$


that is

$$
\begin{cases}\operatorname{div} \mathbb{T}_{a}=0 & \mathscr{L}^{2}-\text { a.e. in } U, \\ \frac{d}{d s}\left(\sigma_{r} \mathrm{t}_{r}\right)-\left[\mathbb{T}_{a}\right] \mathrm{n}_{r}=0 & \mathscr{H}^{1}-\text { a.e. on } C_{r}, \\ \frac{d}{d s}\left(\sigma_{u} \mathrm{t}_{u}\right)-\left[\mathbb{T}_{a}\right] \mathrm{n}_{u}=0 & \mathscr{H}^{1}-\text { a.e. on } C_{u}, \\ {\left[\sigma_{r}\right] \mathrm{t}_{r}+\left[\sigma_{u} \mathrm{t}_{u}\right]=0} & \text { in } c_{1}, c_{2}, \\ \mathbb{T}_{a} \mathrm{~m}=-p_{0} \overline{\mathrm{e}}_{2} & \mathscr{H}^{1}-\text { a.e. on } \Gamma, \\ \mathbb{T}_{a} \mathrm{~m}=0 & \mathscr{H}^{1}-\text { a.e. on } \partial U \backslash\left(\Gamma \cup V_{1} \cup V_{2}\right), \\ \sigma_{r} \mathrm{t}_{r}\left(e_{2}\right)=\sigma_{r} \mathrm{t}_{r}\left(e_{1}\right)=0, & \\ \mathbb{T}_{a} \mathrm{~m} \mathscr{H}^{1}\left\lfloor V_{1} \cup V_{2}+\sigma_{u} \mathrm{t}_{u}\left(\delta_{h_{2}}-\delta_{h_{1}}\right)=\Phi\left\lfloor V_{1} \cup V_{2} .\right.\right.\end{cases}
$$

Let us remark that the last equation is satisfied if the constraint is able to produce a reaction $\Phi\left\lfloor V_{1} \cup V_{2}\right.$ as prescribed by the left-hand side and we assume, from now on, that this is true whenever $h_{1}, h_{2} \in V_{1} \cup V_{2}$. If, on the contrary, $h_{i} \notin V_{1} \cup V_{2}, i=1,2$, then the equation is satisfied only if $\sigma_{u}\left(h_{i}\right)=0$.

Since the geometry of the domain and the applied loads are symmetric with rispect to the axis $x=0$, we look for solutions with the same kind of symmetry. In particular, this implies that if $h_{1} \in V_{1}$ then $h_{2} \in V_{2}$. Moreover, the unknown curve $C_{u}$ must be union of three smooth curves $C_{1}, C_{2}$ and $C_{3}$ connecting the points $h_{1}, c_{1}, c_{2}$ and $h_{2}$ as in Figure $4, C_{2}$ must be symmetric with respect to the axis $x=0$ and $C_{3}$ must be obtained by reflecting $C_{1}$ through to the axis $y$.

Denoting by $a \in(0, h)$ the distance between the reinforcing line $C_{r}$ and the bottom of $U$, and by $\mu \in[0, b / 2)$ the distance of the points $c_{1}$ and $c_{2}$ from the axis $y$, we have that $c_{1}=(-\mu, a)$ and $c_{2}=(\mu, a)$.

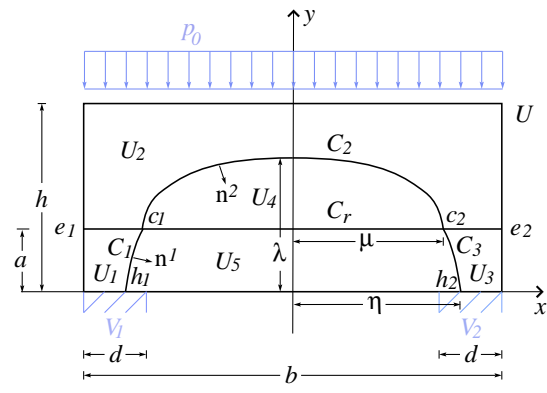

Figure 4: the loaded system

For computational convenience we look for solutions $C_{i}$ that are graphics 
with parametric representations

$$
\begin{aligned}
& C_{1}=\left\{\left(w_{1}(y), y\right): y \in[0, a]\right\}, \quad w_{1} \in C^{1}([0, a]), \\
& C_{2}=\left\{\left(x, w_{2}(x)\right): x \in[-\mu, \mu]\right\}, \quad w_{2} \in C^{1}([-\mu, \mu]), \\
& C_{3}=\left\{\left(-w_{1}(y), y\right): y \in[0, a]\right\} .
\end{aligned}
$$

Of course $C_{r}=\{(x, a): x \in[-b / 2, b / 2]\}$.

With $J_{i}:=\sqrt{1+\left|w_{i}^{\prime}\right|^{2}}$, the tangent unit vectors to $C_{1}, C_{2}$ and $C_{r}$ are, respectively, $\mathrm{t}^{1}=J_{1}^{-1}\left(w_{1}^{\prime} \overline{\mathrm{e}}_{1}+\overline{\mathrm{e}}_{2}\right), \mathrm{t}^{2}=J_{2}^{-1}\left(\overline{\mathrm{e}}_{1}+w_{2}^{\prime} \overline{\mathrm{e}}_{2}\right)$, and $\mathrm{t}_{r}=(1,0)$. Then we have $\mathrm{n}^{1}=J_{1}^{-1}\left(\overline{\mathrm{e}}_{1}-w_{1}^{\prime} \overline{\mathrm{e}}_{2}\right)$ and $\mathrm{n}^{2}=J_{2}^{-1}\left(w_{2}^{\prime} \overline{\mathrm{e}}_{1}-\overline{\mathrm{e}}_{2}\right)$.

Following the ideas of [8], we observe that the first, the fifth and the sixth equations in (13) are satisfied if $\mathbb{T}_{a}$ is given by

$$
\mathbb{T}_{a}:= \begin{cases}-p_{0} \overline{\mathrm{e}}_{2} \otimes \overline{\mathrm{e}}_{2} & \text { in } U_{1} \cup U_{2} \cup U_{3}, \\ 0 & \text { in } U_{4} \cup U_{5} .\end{cases}
$$

We now study the remaining equations in (13).

Second and seventh equation. Since $\left[T_{a}\right]=0 \mathscr{H}^{1}$-a.e. across $C_{r}$ and since $\mathrm{t}_{r}$ is a constant vector, then the second equation is equivalent to

$$
\sigma_{r}=\text { locally constant on } C_{r} \backslash\left\{c_{1}, c_{2}\right\} \text {. }
$$

Using the boundary conditions prescribed by the seventh equation $\sigma_{r} t_{r}\left(e_{2}\right)=$ $\sigma_{r} \mathrm{t}_{r}\left(e_{1}\right)=0$ then we conclude that

Proposition 1. $\sigma_{r}$ is zero on the segments $\overline{e_{1} c_{1}}$ and $\overline{c_{2} e_{2}}$ and is equal to a constant $\bar{\sigma}_{r}$ on the segment $\overline{c_{1} c_{2}}$.

Third equation. On the curves $C_{1}$ and $C_{2}$, by (10), the third equation writes

$$
\begin{cases}\frac{d}{d s}\left(\sigma_{1} \mathrm{t}^{1}\right)-\left(\mathbb{T}_{a}^{1}-\mathbb{T}_{a}^{5}\right) \mathrm{n}^{1}=0 & \text { on } C_{1}, \\ \frac{d}{d s}\left(\sigma_{2} \mathrm{t}^{2}\right)-\left(\mathbb{T}_{a}^{2}-\mathbb{T}_{a}^{4}\right) \mathrm{n}^{2}=0 & \text { on } C_{2},\end{cases}
$$

where $\sigma_{\alpha}=\sigma_{u}\left\lfloor C_{\alpha}\right.$. From (15) and the explicit form of the normals, we find

$$
\left(\mathbb{T}_{a}^{1}-\mathbb{T}_{a}^{5}\right) \mathrm{n}^{1}=J_{1}^{-1} p_{0} w_{1}^{\prime} \overline{\mathrm{e}}_{2}, \quad\left(\mathbb{T}_{a}^{2}-\mathbb{T}_{a}^{4}\right) \mathrm{n}^{2}=J_{2}^{-1} p_{0} \overline{\mathrm{e}}_{2},
$$

and

$$
\frac{d}{d s}\left(\sigma_{\alpha} \mathrm{t}^{\alpha}\right)=J_{\alpha}^{-1} \frac{d}{d x}\left(\sigma_{\alpha} \mathrm{t}^{\alpha}\right)
$$

Setting

$$
\beta_{\alpha}:=\frac{\sigma_{\alpha}}{J_{\alpha}}, \quad \alpha=1,2
$$


the third equation rewrites as

$$
\left\{\begin{array}{l}
\left(\beta_{1} w_{1}^{\prime}\right)^{\prime}=0 \\
\beta_{1}^{\prime}-p_{0} w_{1}^{\prime}=0 \\
\beta_{2}^{\prime}=0 \\
\left(\beta_{2} w_{2}^{\prime}\right)^{\prime}-p_{0}=0 \\
\sigma_{1}=\beta_{1} \sqrt{1+\left|w_{1}^{\prime}\right|^{2}} \\
\sigma_{2}=\beta_{2} \sqrt{1+\left|w_{2}^{\prime}\right|^{2}}
\end{array}\right.
$$

Fourth equation. The fourth equation in the point $c_{1}$ is

$$
\left[\sigma_{r}\right] \mathrm{t}_{r}\left(c_{1}\right)+\left[\sigma_{u} \mathrm{t}_{u}\right]\left(c_{1}\right)=0 .
$$

By Proposition 1 the above equation rewrites

$$
\sigma_{r}\left(c_{1}^{+}\right) \overline{\mathrm{e}}_{1}+\sigma_{u}\left(c_{1}^{+}\right) \mathrm{t}_{u}\left(c_{1}^{+}\right)-\sigma_{u}\left(c_{1}^{-}\right) \mathrm{t}_{u}\left(c_{1}^{-}\right)=0,
$$

that is

$$
\bar{\sigma}_{r} \overline{\mathrm{e}}_{1}+\sigma_{2}(0) \mathrm{t}^{2}(0)-\sigma_{1}(a) \mathrm{t}^{1}(a)=0 .
$$

With the explicit expressions for $\mathrm{t}^{2}(0)$ and $\mathrm{t}^{1}(a)$ we obtain that the fourth equation becomes

$$
\left\{\begin{array}{l}
\bar{\sigma}_{r}+\beta_{2}(-\mu)-\sigma_{1}(a) w_{1}^{\prime}(a)=0, \\
\beta_{2}(-\mu) w_{2}^{\prime}(-\mu)-\beta_{1}(a)=0 .
\end{array}\right.
$$

To the set of differential equations (17) and boundary conditions (18) we can add the additional boundary condition

$$
w_{2}(-\mu)=a .
$$

It is also useful to set

$$
w_{2}(0)=: \lambda>a
$$

and remark that

$$
w_{1}(0)=-\eta, \quad w_{1}(a)=-\mu, \quad w_{2}^{\prime}(0)=0
$$

since $w_{2}$ is a smooth even function. Above $\eta$ denotes the distance of the points $h_{1}$ and $h_{2}$ from the origin.

We now solve for $C_{2}$ and $\sigma_{2}$.

From (17) $)_{3}$ we obtain that $\beta_{2}$ is constant; moreover $\beta_{2} \neq 0$ since otherwise $(17)_{4}$ would imply $p_{0}=0$. Integrating equation $(17)_{4}$ and using $(21)_{3}$ we get

$$
\beta_{2} w_{2}^{\prime}(x)=p_{0} x .
$$

Integrating again and using (20) we obtain

$$
w_{2}(x)=\lambda+\frac{p_{0}}{2 \beta_{2}} x^{2},
$$


and the boundary condition (19) gives

$$
\beta_{2}=-\frac{\mu^{2} p_{0}}{2(\lambda-a)} .
$$

Therefore

$$
w_{2}(x)=\lambda-\frac{\lambda-a}{\mu^{2}} x^{2}, \quad x \in[-\mu, \mu]
$$

and $(17)_{6}$ gives

$$
\sigma_{2}(x)=-p_{0} \sqrt{\frac{\mu^{4}}{4(\lambda-a)^{2}}+x^{2}}, \quad x \in[-\mu, \mu] .
$$

We now solve for $C_{1}$ and $\sigma_{1}$.

Integrating $(17)_{2}$ we get

$$
\beta_{1}-p_{0} w_{1}=c,
$$

with $c$ constant. Using $(18)_{2}$ and $(21)_{2}$ and the expressions of $w_{2}$ and $\sigma_{2}$ computed before we find

$$
c=\beta_{1}(a)-p_{0} w_{1}(a)=\beta_{2} w_{2}^{\prime}(-\mu)+p_{0} \mu=0,
$$

hence

$$
\beta_{1}=p_{0} w_{1} .
$$

With (24), from equation $(17)_{1}$ we obtain

$$
\frac{p_{0}}{2} w_{1}(y)^{2}=k y+d,
$$

where $k$ and $d$ are constants determined by the boundary conditions. In fact, by using $(21)_{1}$ and $(21)_{2}$ we find

$$
k=\frac{p_{0}}{2 a}\left(\mu^{2}-\eta^{2}\right), \quad d=\frac{p_{0}}{2} \eta^{2},
$$

and therefore

$$
w_{1}(y)^{2}=\frac{\mu^{2}-\eta^{2}}{a} y+\eta^{2} .
$$

Since $w_{1}$ must be negative, we get

$$
w_{1}(y)=-\sqrt{\frac{\mu^{2}-\eta^{2}}{a} y+\eta^{2}}, \quad y \in[0, a]
$$

and

$$
\sigma_{1}(y)=-p_{0} \sqrt{\frac{\mu^{2}-\eta^{2}}{a} y+\eta^{2}+\frac{\left(\mu^{2}-\eta^{2}\right)^{2}}{4 a^{2}}}, y \in[0, a] .
$$


Remark 1. Since $w_{1}^{\prime \prime}>0$ then $w_{1}$ is convex.

Remark 2. Since $\sigma_{1}(0)<0$ we have that $h_{1} \in V_{1}$ (hence $h_{2} \in V_{2}$ ), otherwise, as already remarked after (13), the last equation in (17) cannot be satisfied.

Finally, by $(18)_{1}$ we have

$$
\begin{aligned}
\bar{\sigma}_{r} & =\sigma_{1}(a) w_{1}^{\prime}(a)-\beta_{2} \\
& =\frac{p_{0}}{2}\left(\frac{\mu^{2}-\eta^{2}}{2 a^{2} \mu^{2}} \sqrt{4 a^{2}\left(\mu^{2}+\eta^{2}\right)+\left(\mu^{2}-\eta^{2}\right)^{2}}+\frac{\mu^{2}}{\lambda-a}\right) .
\end{aligned}
$$

The previous analysis delivers a family of curves depending on three parameters $\mu, \lambda$ and $\eta$. Some curves of this family for a fixed $\mu$ are depicted in Figure 5 .

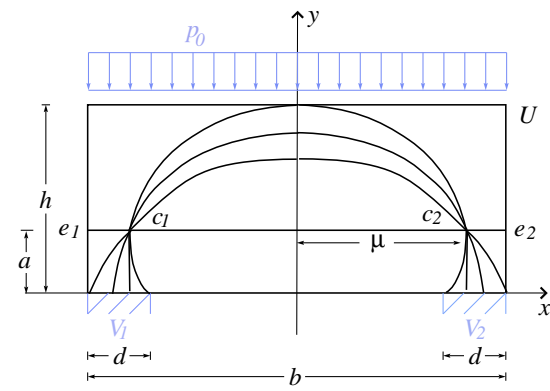

Figure 5: some singular curves for fixed $\mu$

We notice that the curves $C_{1}$ and $C_{3}$ can be either convex or concave. In Figure 6 are depicted the line tensions in the bulk material in both cases. According to (15), in Case 1 we have a tension $-p_{0}$ also in the region delimited by the points $a, b$ and $c$; even if the solution we found is equilibrated, from a physical point of view in such a region it would be more natural to take $\mathbb{T}_{a}=0$. This assumption, though, does not fit with our requirement that $\mathbb{T}_{a}$ is smooth in $U \backslash\left(C_{u} \cup C_{r}\right)$. We therefore believe that, physically, the solution depicted in Case 2 should be preferred.

Hereafter, we call optimal singular curve the curve that minimizes the maximum stress in the no-tension material. According to (23) and (26), the maximum in the curve $C_{2}$ is given by

$$
\max \sigma_{2}=-p_{0} \sqrt{\frac{\mu^{4}}{4(\lambda-a)^{2}}+\mu^{2}},
$$




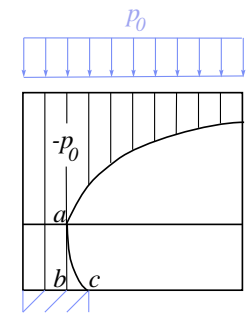

Case 1

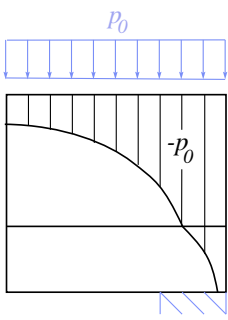

Case 2

Figure 6: line tensions for different curves $C_{1}$ and $C_{3}$

while the maximum in the curve $C_{1}$ is

$$
\max \sigma_{1}= \begin{cases}-p_{0} \sqrt{\frac{\mu^{2}-\eta^{2}}{a} a+\eta^{2}+\frac{\left(\mu^{2}-\eta^{2}\right)^{2}}{4 a^{2}}} & \text { if } \mu>\eta, \\ -p_{0} \sqrt{\eta^{2}+\frac{\left(\mu^{2}-\eta^{2}\right)^{2}}{4 a^{2}}} & \text { if } \mu \leq \eta .\end{cases}
$$

Since to find the optimal singular curve we have to minimize the above maximum stresses, it is convenient to take $\mu \leq \eta$ and $\lambda=h$. Thus, the optimal singular curve is defined for $\lambda=h$ and for $\mu$ and $\eta$ that minimize the function

$$
f(\mu, \eta):=\max \left\{f_{1}(\mu, \eta), f_{2}(\mu)\right\}
$$

with

$$
\begin{aligned}
f_{1}(\mu, \eta) & :=-p_{0} \sqrt{\eta^{2}+\frac{\left(\mu^{2}-\eta^{2}\right)^{2}}{4 a^{2}}}, \\
f_{2}(\mu) & :=-p_{0} \sqrt{\frac{\mu^{4}}{4(h-a)^{2}}+\mu^{2}},
\end{aligned}
$$

on the set

$$
D:=\left\{(\mu, \eta) \in \mathbb{R}^{2}: 0 \leq \mu \leq \eta, b / 2-d \leq \eta \leq b / 2\right\} .
$$

To write $f$ explicitly we study the sign of the function $g(\mu, \eta):=f_{1}(\mu, \eta)-$ $f_{2}(\mu)$. We find that $g(\mu, \eta)=0$ on a monotone increasing curve $\mu=h(\eta)$ intersecting the segments $(0, b / 2-d) \times\{b / 2-d\}$ and $(0, b / 2) \times\{b / 2\}$ as depicted in Figure 7. On the left of the curve $\mu=h(\eta)$ the function $f$ is equal to $f_{1}$ while on the right it is equal to $f_{2}$. A direct computation shows that the minimum is achieved in the point denoted by $A$ in Figure 7 . The minimum is therefore achieved for $\eta=b / 2-d$ and for $\mu$ strictly less than $\eta$.

Of course, as $a$ approaches zero, $\mu$ approaches $\eta$.

The optimal singular curve, which is represented in Figure 8, resembles the line tensions found experimentally on reinforced concrete beams, see Park and Paulay [13, Figure 7.8]. 


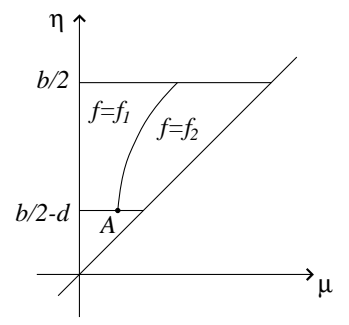

Figure 7: the domain $D$

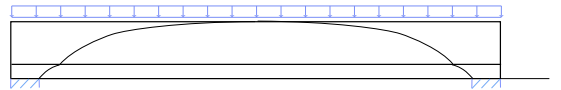

Figure 8: the optimal singular curve for a slender panel

\section{Conclusions}

A theory for panels of no-tension material with a reinforcing tensile resistant unidimensional material has been put forward. Following the work by Lucchesi, Silhavy and Zani [8], we have allowed the stresses to be singular, in that they may concentrate on curves. The stress field in the no-tension material has been assumed to be negative semi-definite, while on the reinforcement the stress has been taken to be positive. In particular, the equilibrium equations for a rectangular panel loaded on the top by a uniform vertical load and with an horizontal reinforcement have been studied and solved. The family of solutions that we have found can be parametrized by means of singular curves where the stress in the no-tension material concentrates. Among these curves we determine the curve that minimizes the maximum stress: this singular curve resembles the line tensions found experimentally on reinforced concrete beams.

[1] M. Angelillo, E. Babilio, A. Fortunato, Singular stress fields for masonrylike vaults. Continuum Mech. Thermodyn. DOI 10.1007/s00161-012-0270-9.

[2] D. Bigoni, G. Noselli, Localized stress percolation through dry masonry walls. Part I - Experiments. European Journal of Mechanics, A/Solids 29 2010, n. 3, 291-298.

[3] D. Bigoni, G. Noselli, Localized stress percolation through dry masonry walls. Part II - Modelling. European Journal of Mechanics, A/Solids 29 2010, n. 3, 299-307.

[4] M. Degiovanni, A. Marzocchi, A. Musesti, Cauchy fluxes associated with tensor fields having divergence measure. Arch. Ration. Mech. Anal. 147 (1999), n. 3, 197-223. 
[5] G. Del Piero, Constitutive equation and compatibility of the external loads for linear elastic masonry-like materials. Meccanica 24 (1989), n. 3, 150162.

[6] G. Del Piero, Limit analysis and no-tension materials. Int. J. Plasticity 14 (1998), n. 1-3, 150-162.

[7] M. Giaquinta, E. Giusti, Researches on the equilibrium of masonry structures. Arch. Rational Mech. Anal., 88 (1985), n. 4, 359-392.

[8] M. Lucchesi, M. Šilhavý, N. Zani, A new class of equilibrated stress fields for no-tension bodies. Journal of mechanics of materials and structures $\mathbf{1}$ (2006), n. 3, 503-539.

[9] M. Lucchesi, M. Šilhavý, N. Zani, A note on equilibrated stress fields for notension bodies under gravity. Quart. Appl. Math. 66 (2007), 605-624.

[10] M. Lucchesi, M. Šilhavý, N. Zani, Equilibrated divergence measure stress tensor fields for heavy masonry bodies. European Journal of Mechanics A/Solids 28 (2009), 223-232.

[11] M. Lucchesi, M. Šilhavý, N. Zani, On the balance equation for stresses concentrated on curves. J. Elasticity 90 (2008), n. 2, 209-223.

[12] M. Lucchesi, M. Šilhavý, N. Zani, Integration of measures and admissible stress fields for masonry bodies. J. Mech. Mater. Struct. 3 (2008), 675696.

[13] R. Park, T. Paulay, Reinforced concrete structures. John Wiley and Sons, 1975.

[14] P. Podio-Guidugli, Examples of concentrated contact interactions in simple bodies. J. Elasticity 75 (2004), n. 2, 167-186. 